\title{
Efficacy of celecoxib for acute pain management following total hip arthroplasty in elderly patients: A prospective, randomized, placebo-control trial
}

\author{
JIA CHEN，WEI ZHU，ZHENXIANG ZHANG，LIXIAN ZHU，WENJIE ZHANG and YAQING DU
}

Orthopedic Department, The Affiliated Taizhou People's Hospital of Nantong University, Taizhou, Jiangsu 225300, P.R. China

Received August 11, 2014; Accepted May 1, 2015

DOI: $10.3892 / \mathrm{etm} .2015 .2512$

\begin{abstract}
The aim of the present study was to determine whether celecoxib is able to ameliorate pain intensity, provide a narcotic-sparing effect, achieve early ambulation and improve rehabilitation following total hip arthroplasty (THA) in elderly patients. Peri- and post-operative oral celecoxib was administered to verify the efficacy of celecoxib for acute pain management in a multimodal analgesic strategy. All 64 eligible patients were randomly allocated to either the celecoxib group, who took an oral $400 \mathrm{mg}$ capsule of celecoxib peri-operatively and $200 \mathrm{mg}$ per $12 \mathrm{~h}$ post-operatively for the first 5 days, or the control group, who were orally treated with a placebo capsule having the same appearance. A multimodal analgesic technique was used in which oral celecoxib or placebo capsule was combined with intravenous patient-controlled analgesia (PCA) morphine pump for peri- and post-operative pain management. Pain assessments were recorded at 6, 12, 24, 48 and $72 \mathrm{~h}$, and 7 and 14 days after THA using the visual analog scale (VAS). PCA morphine consumption; 6, 12, 24 and 48-h post-operative Harris hip score (HHS); time interval until initial ambulation; rates of urinary retention and post-operative nausea and vomiting (PONV) within $72 \mathrm{~h}$; and intra- and post-operative blood loss were also documented. The celecoxib and control groups comprised 34 and 30 patients, respectively. Baseline demographics were comparable between the two groups. The post-operative VAS in the celecoxib group was significantly lower than that in the control group at 12, 24, 48 and $72 \mathrm{~h}$ after THA. The post-operative HHS had no significant difference
\end{abstract}

Correspondence to: Dr Jia Chen, Orthopedic Department, The Affiliated Taizhou People's Hospital of Nantong University, 210 Yingchun Road, Taizhou, Jiangsu 225300, P.R. China

E-mail: mike20111201@hotmail.com

Abbreviations: PCA, patient-controlled analgesia; THA, total hip arthroplasty; VAS, visual analog scale; HHS, Harris hip score; PONV, postoperative nausea and vomiting; NSAIDs, non-steroidal anti-inflammatory drugs; COX, cyclooxygenase; PG, prostaglandin; $\mathrm{CV}$, cardiovascular

Key words: celecoxib, total hip arthroplasty, pain management between the two groups, while the time interval until initial ambulation in the celecoxib group (4.5 \pm 1.2 days) was significantly less than that in the control group (5.83 \pm 2.04 days; $\mathrm{P}<0.05)$. Morphine consumption was significantly decreased in the celecoxib group when compared with the control group at $6,12,24$ and $24 \mathrm{~h}$. Although the 72-h post-operative rates of urinary retention and PONV were lower in the celecoxib group than in the control group, there were no significant differences in these rates between the two groups. The intraor post-operative blood loss was not significantly different between groups. In conclusion, pre-and post-operative oral celecoxib in a multimodal analgesic strategy can achieve favorable pain relief, reduce opioid consumption, and provide earlier ambulation and improved rehabilitation when compared with PCA morphine alone following THA in elderly patients.

\section{Introduction}

Total hip arthroplasty (THA) is an effective therapeutic intervention for pain relief in elderly patients with hip degeneration (1). Although the treatment can radically resolve the chronic pain that results from hip lesions, the acute pain derived from hip surgery continues to afflict patients post-operatively, particularly in elderly patients, and this may cause physiological and psychological complications $(2,3)$. Unrelieved post-operative pain in elderly individuals may lead to delayed mobilization and rehabilitation, poor surgical outcomes and prolonged hospital stay $(4,5)$. Consequently, there is a clear requirement for post-operative pain relief that is able to not only reduce pain-related complications but also achieve rapid rehabilitation following THA.

Non-steroidal anti-inflammatory drugs (NSAIDs) are universally utilized as an assistant approach to acute pain management (6). However, due to non-selective inhibitory effects on cyclooxygenase (COX)-1 and COX-2, several side-effects of conventional NSAIDs have emerged (7). Celecoxib, as a representative of the class of highly selective COX-2-specific inhibitors, can specifically inhibit the functioning of COX-2 in vivo without affecting the protective action of COX-1. In addition, it suppresses the synthesis of prostaglandins (PGs) and thereby reduces their effects, which include reduction of the pain threshold and enhancement of the transduction and transmission of nociceptive information in the central and peripheral nociceptors (8). Several studies (9-11) 
have reported the use of celecoxib as a pre-emptive approach to realize satisfactory peri- or post-operative clinical pain management.

Patient-controlled opioid analgesia pumps have commonly been used for post-operative acute pain management. However, certain complications, such as post-operative nausea and vomiting (PONV) and urinary retention, are attributable to the usage of opioid narcotic when given at increasing concentrations (12). In order to achieve a compromise that not only decreases opioid consumption but also achieves favorable pain relief, a combination of COX-2 inhibitor and patient-controlled analgesia (PCA) may be administered.

In the Affiliated Taizhou People's Hospital of Nantong University (Taizhou, China), a team has focused on studying the efficacy of celecoxib following hip arthroscopic surgery (13). However, there have been few studies evaluating the use of oral celecoxib in the elderly population undergoing THA when combined with PCA post-operatively in clinical practice. The aim of the present study was to evaluate the efficacy of celecoxib following THA in a multimodal analgesic strategy. The hypothesis that peri-operative pain management using oral celecoxib in combination with PCA can improve pain intensity, achieve a narcotic-sparing effect, early ambulation, and better rehabilitation after THA in elderly patients was investigated.

\section{Patients and methods}

Patient selection. Between June 2011 and June 2013, a total of 64 patients underwent THA in the Affiliated Taizhou People's Hospital of Nantong University were considered eligible to participate in the prospective randomized placebo-controlled study. Informed consent was obtained from all patients who participated in this study. The study was approved by the Regional Ethics Committee of Taizhou People's Hospital.

Inclusion and exclusion criteria. Patients who were $>65$ years old, with osteoarthritis and aseptic necrosis of femoral head met the inclusion criteria. The exclusion criteria for this study were allergy to celecoxib, sulfa drugs, acetylsalicylic acid or NSAIDs; existence of blood coagulation dysfunction, or severe hepatic and renal dysfunction; evidence of active peptic ulcer or recent cardio-cerebrovascular events; relevant contraindications of THA, such as active or chronic infection in the target hip or other region of the body and chronic osteomyelitis; active joint tuberculosis or blood disease; and inability to undergo lumbar anesthesia due to previous lumbar diseases or surgeries. Patients who experienced acute injury were excluded in order to establish a unified group of patients that were in good physiological and psychological condition prior to the surgery. Patients who refused to anticipate in the study or who had inadequate training for PCA usage were also excluded.

Study design and procedure. A total of 64 consecutive patients who met the requirements of the study were given sealed, opaque and consecutively numbered envelopes, and then randomly allocated to either the celecoxib or the control group by means of a randomly generated number pattern that had been designed prior to recruitment. The anesthesiologist and surgeon caring for the patient were blind to the patient's group assignment and outcome data. All relevant data were documented by an independent observing surgeon (Jia Chen). The posterolateral approach between 10 and $14 \mathrm{~cm}$ was used for all procedures with repair of the capsule and external rotators. Press-fit components (Pinnacle; DePuy Synthes, Warsaw, IN, USA), and cement-free hydroxyapatite-coated stems (Corail; DePuy Synthes) were available for THA in the present study.

All surgeries were performed under combined spinal and epidural anesthetics. Patients in the study accepted a multimodal analgesic technique, which comprised the use of oral celecoxib (Celebrex; Pfizer, Inc., New York, NY, USA) or placebo capsule combined with an intravenous PCA morphine pump (ACE Medical Industry Co., Ltd., Goyang, Korea) for peri-operative and post-operative pain management. A PCA morphine $(1 \mathrm{mg} / \mathrm{ml})$ pump was set to deliver a $2-\mathrm{ml}$ bolus dose with a lockout interval of $10 \mathrm{~min}$. The total amount of morphine consumption was recorded. An experienced nurse (Yaqing Du) had trained all patients how to use the PCA device prior to surgery.

The celecoxib group received an initial 400-mg dose $1 \mathrm{~h}$ pre-operatively, and a $200 \mathrm{mg}$ dose was given each $12 \mathrm{~h}$ post-operatively for the first 5 days. Similarly, the control group took a capsule of the same appearance at identical intervals pre- and post-operatively.

Outcome assessments. Baseline demographic variables including the number of patients, mean age, proportion of each gender, body mass index (BMI) and surgery duration in each group are shown in Table I. The primary outcome was pain score, as measured by a visual analog scale (VAS) of 0-10 (with 0 representing no pain and 10 representing the worst pain imaginable). The secondary outcomes included PCA morphine consumption; post-operative Harris hip score (HHS); time interval until initial ambulation; rates of urinary retention and PONV within $72 \mathrm{~h}$; and intra- and post-operative blood loss. Pain assessments were conducted at 6,12, 24,48 and $72 \mathrm{~h}$, and 7 and 14 days after THA. The amounts of PCA morphine consumption were recorded by an independent observer at 6 , 12, 24 and $48 \mathrm{~h}$ post-operatively. Patients were encouraged to ambulate with a walker or two crutches as soon as possible and the time when patients initially ambulated was recorded. The HHS at 3, 7 and 14 days was also documented. All patients received 5,000 IU low-molecular-weight heparin subcutaneously daily for prophylaxis of thromboembolism in the first 7 days post-operatively.

Statistical analysis. Demographic variables, post-operative HHS and intra-/post-operative blood loss were analyzed by independent samples t-test. Rates of urinary retention and PONV were analyzed by Chi-square test. Pain scores, morphine consumption and time interval until initial ambulation were analyzed by Mann-Whitney U test. Statistical analysis was performed using SPSS software, version 19.0 (SPSS, Inc., Chicago, IL, USA). $\mathrm{P}<0.05$ was considered to indicate a statistically significant difference.

\section{Results}

Baseline demographics and pain assessments. There were no significant differences between the two groups in terms 
Table I. Patient demographics and baseline characteristics.

\begin{tabular}{lccc}
\hline Variable & Celecoxib group & Control group & P-value \\
\hline Number of patients & 34 & 30 & - \\
Age $($ years) & $73.6 \pm 4.8$ & $74.5 \pm 4.5$ & $>0.05$ \\
Male/female & $16 / 18$ & $13 / 17$ & - \\
BMI $\left(\mathrm{kg} / \mathrm{m}^{2}\right)^{\mathrm{a}}$ & $32.3 \pm 5.6$ & $34.4 \pm 6.2$ & $>0.05$ \\
Surgery duration $(\mathrm{min})^{\mathrm{a}}$ & $98.2 \pm 13.7$ & $96.5 \pm 14.9$ & $>0.05$
\end{tabular}

BMI, body mass index. ${ }^{a}$ Values are mean \pm standard deviation.

Table II. Intraoperative and postoperative data.

\begin{tabular}{lccc} 
Variable & Celecoxib group & Control group & P-value \\
\hline Intra-operative blood loss (ml) & $432.4 \pm 62.1$ & $458.0 \pm 67.6$ & 0.119 \\
Post-operative blood loss (ml) & $233.7 \pm 25.9$ & $242.3 \pm 27.5$ & 0.201 \\
HHS (time of ambulation) & $77.6 \pm 4.3$ & $75.5 \pm 5.2$ & $79.4 \pm 4.6$ \\
HHS (7 days) & $81.5 \pm 5.1$ & $83.9 \pm 5.1$ & 0.081 \\
HHS (14 days) & $86.1 \pm 4.7$ & $5.83 \pm 2.04$ & 30 \\
Time interval until first ambulation (days) & $4.5 \pm 1.2$ & 47 \\
Urinary retention rates (\%) & 23.5 & 0.008 \\
PONV rates (\%) & 35.3 & 0.559 \\
\hline
\end{tabular}

HHS, Harris hip score; PONV, postoperative nausea and vomiting. Values are mean \pm standard deviation unless otherwise indicated.

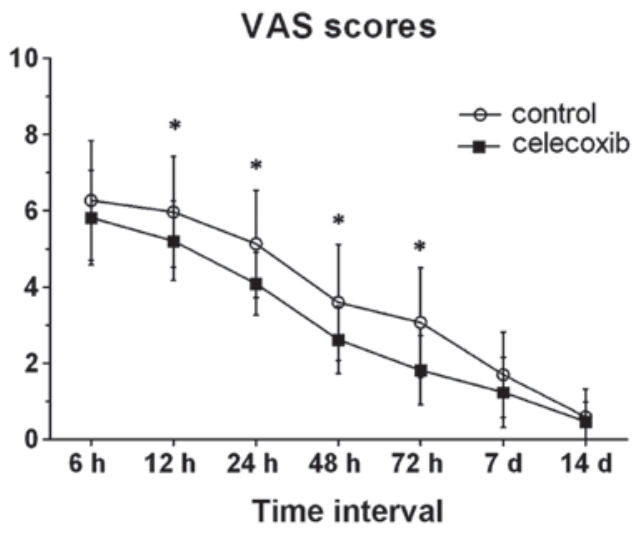

Figure 1. Postoperative pain scores. ${ }^{*} \mathrm{P}<0.05$ compared with the celecoxib group. The post-operative VAS pain score was significantly lower at $12,24,48$ and $72 \mathrm{~h}$ in the celecoxib group compared with the control group. VAS, visual analog scale.

of the number of patients, gender distribution, age, BMI and duration of surgery (Table I). In comparison with the control group, the post-operative VAS pain score was significantly lower at 12, 24, 48 and $72 \mathrm{~h}$ after THA in the celecoxib group. When ambulating and accessing rehabilitation until discharge, the two groups had no statistically significant differences between them with regard to post-operative VAS scores ( 7 days, $\mathrm{P}=0.105 ; 14$ days, $\mathrm{P}=0.648$ ), although patients in the two groups had a decreasing perception of pain (Fig. 1).

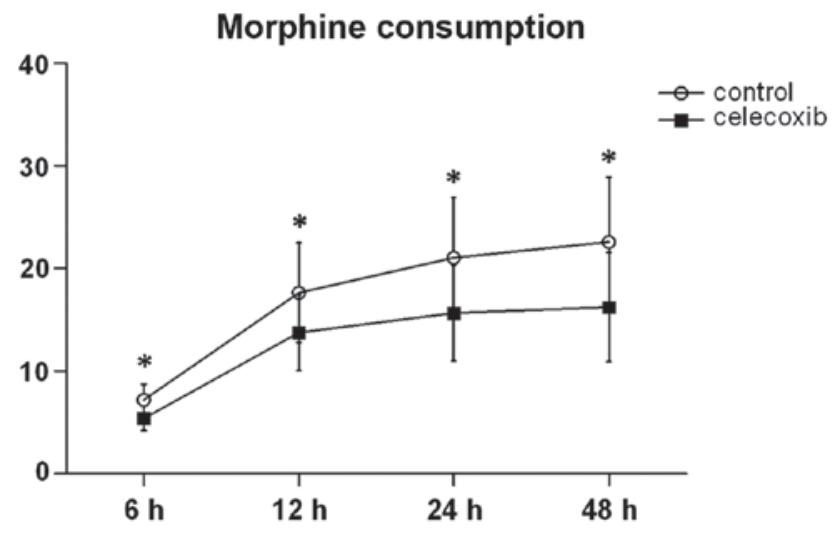

Figure 2. PCA morphine consumption. ${ }^{*} \mathrm{P}<0.05$ compared with the celecoxib group. PCA morphine consumption was significantly decreased in the celecoxib group compared with the control group at 6,12,24 and $24 \mathrm{~h}$ postoperatively. PCA, patient-controlled analgesia.

HHS results and time of initial ambulation. The post-operative HHS did not significantly differ between the two groups at the time of ambulation and at 7 and 14 days after THA $(\mathrm{P}=0.081$, 0.080 and 0.071 , respectively; Table II). The time interval until initial ambulation in the celecoxib group (4.5 \pm 1.2 days) was significantly less than that in the control group (5.83 \pm 2.04 days; $\mathrm{P}<0.05$; Table II).

Opioid usage and associated complications. PCA morphine consumption was significantly decreased in the celecoxib 
group when compared with the control group at 6, 12, 24 and $24 \mathrm{~h}$ (Fig. 2). Within $72 \mathrm{~h}$ after the surgery, the rates of urinary retention were 30 and $23.5 \%$ in the control and celecoxib groups, respectively. Post-operative PONV rates were $47 \%$ in the control group and $35.3 \%$ in the celecoxib group (Table II). The data revealed no statistically significant difference between the two groups in PONV rates $(\mathrm{P}=0.355)$; similar results were exhibited in urinary retention rates in the two groups $(\mathrm{P}=0.559)$. The intra- or post-operative blood loss also showed no statistically significant difference between groups (Table II).

\section{Discussion}

This prospective randomized study demonstrates that using oral celecoxib pre-emptively and post-operatively can achieve effective clinical outcomes when compared with placebo for acute pain management after THA in elderly patients. Celecoxib can improve pain management, and the quality and function of post-operative recovery, in addition to providing narcotic-sparing effects in a multimodal analgesic strategy. Few side-effects were found in the short time interval that was observed.

Several studies have applied celecoxib for acute pain management in peri-operative multimodal analgesic strategies (13-15). A previous study conducted in our institution (13) demonstrated that $200 \mathrm{mg}$ celecoxib administered in one dose pre-operatively was effective in reducing post-operative pain and provided a narcotic-sparing effect in patients undergoing arthroscopic hip surgery. Reuben et al (15) studied the peri-operative dose of celecoxib in pain management following spinal fusion surgery, and concluded that an oral 400-mg celecoxib capsule as pre-emptive analgesia followed by an additional $200 \mathrm{mg}$ dose $12 \mathrm{~h}$ later resulted in a $31 \%$ reduction in morphine use and significantly lower pain scores when compared with a pre-operative $200 \mathrm{mg}$ dose, in which there was only a $9 \%$ reduction in morphine use. Although these studies studied different celecoxib doses, there were no differences in peri- and post-operative complications associated with bleeding, wound hematoma and gastrointestinal reactions between them. In the present study, oral $400 \mathrm{mg}$ celecoxib was administered pre-operatively followed by $200 \mathrm{mg}$ per $12 \mathrm{~h}$ post-operatively in combination with intravenous PCA morphine pump for the first 5 days and this demonstrated the favorable efficacy of celecoxib in acute pain management following THA. Thus, it is considered that celecoxib can take an important role in multimodal analgesic regimens.

There have been a number of studies demonstrating that COX-2 inhibitors can contribute to reduced VAS pain scores in peri- and post-operative pain management following total joint arthroplasty $(9,14,16)$, and a meta-analysis (17) demonstrated that reasonably used COX-2 inhibitors can reduce post-operative pain and VAS scores when compared with placebo. In the current study, the peri-operative VAS scores were significant lower in the celecoxib group at 12,24,48 and $72 \mathrm{~h}$ after THA. When celecoxib usage was stopped after the initial 5-day therapeutic period, the two groups in the study had no significant difference in VAS scores. In addition, the elderly patients in the celecoxib group experienced an episode of mild pain relief, which might minimize acute pain-related complication, such as delirium, hyperpiesia and neuropsychological tension $(18,19)$.

The quality and function of post-operative recovery is an indication of pain management following THA. Patients who were willing to walk or take exercise had less pain perception when ambulating. The post-operative HHS was evaluated at the time of ambulation, and at 7 and 14 days. In addition, the interval from surgery until initial ambulation was evaluated. Kang et al (14) supplemented intravenous PCA with pre-emptive $200 \mathrm{mg}$ celecoxib followed by intra-operative periarticular injections and observed no significant difference in early walking activity from patients treated with PCA alone; however, patients treated with the multimodal analgesic regimen using oral celecoxib started walking or taking exercise earlier. In the present study, although the HHSs in the celecoxib group were slightly higher than those in the control group, the scores exhibited no statistically significant differences between the two groups post-operatively until discharge. However, elderly patients in the celecoxib group had a reduced time interval until auxiliary ambulation. Therefore, it can be considered that peri-operative pain management with adjuvant celecoxib can decrease the perception of pain and improve pain relief when ambulating.

There are a variety of peri-operative complications associated with opioid narcotics, such as PONV, urinary retention, pruritus, drowsiness and sedation (20-23). Utilizing celecoxib plus opioid medication is a prevalent approach to multimodal analgesic strategy in acute pain management post-operatively, which can decrease the consumption of opioid. In the present study, the post-operative opioid consumption in the celecoxib group was $38 \%$ less than in control group; however, there were no statistically significant differences in urinary retention rates or PONV rates when compared with the placebo group. Huang et al (9) compared celecoxib with placebo in pain management following total knee arthroplasty, and concluded that peri-operative celecoxib can contribute to a reduction in post-operative opioid consumption of $40 \%$. However, although the reduced opioid use led to a decline in the rate of PONV incidence (28 vs. $43 \%$ ), there were no difference in PONV incidence in the two groups. This observation was consistent with the present study, which showed 35.3 and $47 \%$ PONV rates in the celecoxib and control groups, respectively. No statistically significant difference in PONV rates was identified between the two groups. The rates of urinary retention incidence in the present study showed an analogous effect (23.5 vs. 30\%). As the number of patients in this study was limited, a study with a larger sample size is required to verify the correlation between declined opioid consumption and opioid-related side-effects.

Celecoxib, as a representative of the class of selective COX-2 inhibitors, has the advantage of selective effects on COX-2 when compared with conventional NSAIDs. To the best of our knowledge, several studies have demonstrated an association between NSAIDs and the functioning of platelets (24-28). Selective COX-2 inhibitors, however, have no ability to inhibit platelet aggregation or coagulation function (29-31). They specifically inhibit the effect of COX-2, which takes an important role in catalyzing PG synthesis and the subsequent inflammatory response (32). Thus, COX-2 inhibitors avoid the side-effects of NSAIDs, which are primarily actualized by the inhibition of COX-1, resulting in gastrointestinal adverse 
reactions, the inhibition of platelet aggregation and impairment of renal function. Ekman et al (10) administered oral $400 \mathrm{mg}$ celecoxib $1 \mathrm{~h}$ prior to arthroscopic knee surgery. The results suggested that celecoxib did not interfere with normal hematologic function. A retrospective cohort study (33) based on databases covering $>1.3$ million patients aged $>66$ years demonstrated lower rates of upper gastrointestinal hemorrhage for celecoxib in comparison with conventional NSAIDs. In the present study, the volumes of peri- and post-operative blood loss had no significant difference between the two observed groups, and no antiplatelet activity-related complications, such as incision hematoma, bleeding and ulcer bleeding, were exhibited in the observed interval. The period of acute pain management is relatively short, and the observation of the side-effects of celecoxib associated with the inhibition of platelet function may require a prolonged observation period.

There are certain limitations to the present study. First, due to the rigid inclusion and exclusion criteria, there were limited numbers of patients who were eligible for this study; this resulted in a small sample size and may have introduced potential selection bias. Secondly, patients who suffered from fresh fracture of the femur neck were excluded, although this is a preferable indication for elderly patients undergoing THA. However, the pain derived from acute hip trauma, post-traumatic transportation and consequent pre-operative treatment, such as limb skeletal traction, may lead to a higher incidence of acute confusional states and greater pain intensity, which may influence the pre-operative psychological state and post-operative recovery $(34,35)$. In order to establish a unified group of patients who were in a good physiological and psychological state prior to the surgery, patients who experienced acute injury were excluded and another study may be conducted to focus on the population with traumatic hip injury. Finally, the safety of celecoxib has not been verified in this study, since the observation period was short. To the best of our knowledge, coxib-type drugs are associated with a potential increased risk for thrombosis, and renal and cardiovascular (CV) adverse events (36-39). The safety of drugs of the coxib class continues to require further evaluation, particularly in elderly patients who are more likely to suffer from CV disease and renal function disorders. Larger observation periods for celecoxib safety verification are planned in our future study.

In conclusion, the present study demonstrates that using celecoxib in a multimodal analgesic strategy pre-and post-operatively can achieve favorable efficacy in the management of pain. The use of a treatment regimen comprising oral treatment with celecoxib at a dose of $400 \mathrm{mg}$ pre-emptively and $200 \mathrm{mg}$ per $12 \mathrm{~h}$ post-operatively in combination with PCA morphine pump should improve pain intensity, reduce opioid consumption, and achieve early ambulation and improved rehabilitation after THA in elderly patients.

\section{References}

1. Kennedy JW, Johnston L, Cochrane L and Boscainos PJ: Outcomes of total hip arthroplasty in the octogenarian population. Surgeon 11: 199-204, 2013.

2. Ferrell BA: Pain management in elderly people. J Am Geriatr Soc 39: 64-73, 1991

3. Eid $\mathrm{T}$ and Bucknall T: Documenting and implementing evidence-based post-operative pain management in older patients with hip fractures. J Orthop Nurs 12: 90-98, 2008.
4. Illgen RL, Pellino TA, Gordon DB, Butts S and Heiner JP: Prospective analysis of a novel long-acting oral opioid analgesic regimen for pain control after total hip and knee arthroplasty. J Arthroplasty 21: 814-820, 2006.

5. Morrison RS, Magaziner J, McLaughlin MA, et al: The impact of post-operative pain on outcomes following hip fracture. Pain 103: 303-311, 2003.

6. Moore ND: In search of an ideal analgesic for common acute pain. Acute Pain 11: 129-137, 2009.

7. Sostres C, Gargallo CJ, Arroyo MT and Lanas A: Adverse effects of non-steroidal anti-inflammatory drugs (NSAIDs, aspirin and coxibs) on upper gastrointestinal tract. Best Pract Res Clin Gastroenterol 24: 121-132, 2010.

8. Burian $\mathrm{M}$ and Geisslinger G: COX-dependent mechanisms involved in the antinociceptive action of NSAIDs at central and peripheral sites. Pharmacol Ther 107: 139-154, 2005.

9. Huang YM, Wang CM, Wang CT, Lin WP, Horng LC and Jiang CC: Perioperative celecoxib administration for pain management after total knee arthroplasty - A randomized, controlled study. BMC Musculoskelet Disord 9: 77, 2008.

10. Ekman EF, Wahba M and Ancona F: Analgesic efficacy of perioperative celecoxib in ambulatory arthroscopic knee surgery: A double-blind, placebo-controlled study. Arthroscopy 22: 635-642, 2006

11. Sinatra R: Role of COX-2 inhibitors in the evolution of acute pain management. J Pain Symptom Manage 24 (Suppl): S18-S27, 2002.

12. Remy C, Marret E and Bonnet F: Effects of acetaminophen on morphine side-effects and consumption after major surgery: Meta-analysis of randomized controlled trials. $\mathrm{Br} \mathrm{J}$ Anaesth 94: 505-513, 2005.

13. Zhang Z, Zhu W, Zhu L and Du Y: Efficacy of celecoxib for pain management after arthroscopic surgery of hip: A prospective randomized placebo-controlled study. Eur J Orthop Surg Traumatol 24: 919-923, 2014

14. Kang H,Ha YC, Kim JY, Woo YC, Lee JS and Jang EC: Effectiveness of multimodal pain management after bipolar hemiarthroplasty for hip fracture: A randomized, controlled study. J Bone Joint Surg Am 95: 291-296, 2013.

15. Reuben SS and Ekman EF: The effect of cyclooxygenase-2 inhibition on analgesia and spinal fusion. J Bone Joint Surg Am 87: 536-542, 2005 .

16. Schroer WC, Diesfeld PJ, LeMarr AR and Reedy ME: Benefits of prolonged postoperative cyclooxygenase-2 inhibitor administration on total knee arthroplasty recovery: A double-blind, placebo-controlled study. J Arthroplasty 26 (Suppl): 2-7, 2011.

17. Lin J,Zhang Land Yang H: Perioperative administration of selective cyclooxygenase- 2 inhibitors for postoperative pain management in patients after total knee arthroplasty. J Arthroplasty 28: 207-213. e2, 2013.

18. RadinovicK,MilanZ,Markovic-DenicL,Dubljanin-RaspopovicE, Jovanovic B and Bumbasirevic V: Predictors of severe pain in the immediate postoperative period in elderly patients following hip fracture surgery. Injury 45: 1246-1250, 2014.

19. Feldt KS and Oh HL: Pain and hip fracture outcomes for older adults. Orthop Nurs 19: 35-44, 2000.

20. Keïta H, Geachan N, Dahmani S, et al: Comparison between patient-controlled analgesia and subcutaneous morphine in elderly patients after total hip replacement. Br J Anaesth 90: 53-57, 2003.

21. Dorr LD, Raya J, Long WT, Boutary M and Sirianni LE: Multimodal analgesia without parenteral narcotics for total knee arthroplasty. J Arthroplasty 23: 502-508, 2008.

22. Hudcova J, McNicol E, Quah C, Lau J and Carr DB: Patient controlled intravenous opioid analgesia versus conventional opioid analgesia for postoperative pain control: A quantitative systematic review. Acute Pain 7: 115-132, 2005.

23. Wheeler M, Oderda GM, Ashburn MA and Lipman AG: Adverse events associated with postoperative opioid analgesia: A systematic review. J Pain 3: 159-180, 2002.

24. Brooks P, Emery P, Evans JF, et al: Interpreting the clinical significance of the differential inhibition of cyclooxygenase-1 and cyclooxygenase-2. Rheumatology (Oxford) 38: 779-788, 1999.

25. Cheng JC, Siegel LB, Katari B, Traynoff SA and Ro JO: Nonsteroidal anti-inflammatory drugs and aspirin: A comparison of the antiplatelet effects. Am J Ther 4: 62-65, 1997.

26. Lanas A, García-Rodríguez LA, Arroyo MT, et al: Investigators of the Asociación Española de Gastroenterología (AEG): Effect of antisecretory drugs and nitrates on the risk of ulcer bleeding associated with nonsteroidal anti-inflammatory drugs, antiplatelet agents, and anticoagulants. Am J Gastroenterol 102: 507-515, 2007. 
27. Carson JL, Strom BL, Soper KA, West SL and Morse ML: The association of nonsteroidal anti-inflammatory drugs with upper gastrointestinal tract bleeding. Arch Intern Med 147: 85-88, 1987.

28. Gladding PA, Webster MW, Farrell HB, Zeng IS, Park R and Ruijne N: The antiplatelet effect of six non-steroidal anti-inflammatory drugs and their pharmacodynamic interaction with aspirin in healthy volunteers. Am J Cardiol 101: 1060-1063, 2008.

29. Leese PT, Hubbard RC, Karim A, Isakson PC, Yu SS and Geis GS: Effects of celecoxib, a novel cyclooxygenase-2 inhibitor, on platelet function in healthy adults: A randomized, controlled trial. J Clin Pharmacol 40: 124-132, 2000.

30. Graff J, Arabmotlagh M, Cheung R, Geisslinger G and Harder S: Effects of parecoxib and dipyrone on platelet aggregation in patients undergoing meniscectomy: A double-blind, randomized, parallel-group study. Clin Ther 29: 438-447, 2007.

31. Leese PT, Talwalker S, Kent JD and Recker DP: Valdecoxib does not impair platelet function. Am J Emerg Med 20: 275-281, 2002.

32. Buttar NS and Wang KK: The 'aspirin' of the new millennium: Cyclooxygenase-2 inhibitors. Mayo Clin Proc 75: 1027-1038, 2000.

33. Mamdani M, Rochon PA, Juurlink DN, et al: Observational study of upper gastrointestinal haemorrhage in elderly patients given selective cyclo-oxygenase-2 inhibitors or conventional non-steroidal anti-inflammatory drugs. BMJ 325: 624, 2002.
34. Johansson I, Bååth C, Wilde-Larsson B and Hall-Lord ML: Acute confusion states, pain, health, functional status and quality of care among patients with hip fracture during hospital stay. Int J Orthop Trauma Nurs 17: 120-130, 2013.

35. Gustafson Y, Berggren D, Brännström B, et al: Acute confusional states in elderly patients treated for femoral neck fracture. J Am Geriatr Soc 36: 525-530, 1988.

36. Shi S and Klotz U: Clinical use and pharmacological properties of selective COX-2 inhibitors. Eur J Clin Pharmacol 64: 233-252, 2008.

37. Bombardier C, Laine L, Reicin A, et al: VIGOR Study Group: Comparison of upper gastrointestinal toxicity of rofecoxib and naproxen in patients with rheumatoid arthritis. N Engl J Med 343: $1520-1528,2000$

38. Brater DC, Harris C, Redfern JS and Gertz BJ: Renal effects of COX-2-selective inhibitors. Am J Nephrol 21: 1-15, 2001.

39. Brater DC: Effects of nonsteroidal anti-inflammatory drugs on renal function: Focus on cyclooxygenase-2-selective inhibition. Am J Med 107 (Suppl 1): 65S-71S, 1999. 